Artigo

\title{
Análise do Índice de Anomalia de Chuva para a Microbacia de Santa Maria/Cambiocó, RJ
}

\author{
Gustavo Carneiro de Noronha ${ }^{1,2}$, Mônica de Aquino Galeano Massera da Hora ${ }^{3}$, \\ Luciene Pimentel da Silva ${ }^{1}$ \\ ${ }^{1}$ Programa de Pós-Graduação em Meio Ambiente, Universidade do Estado do Rio de Janeiro, \\ Rio de Janeiro, RJ, Brasil. \\ ${ }^{2}$ Departamento de Engenharia Agrícola e Meio Ambiente, Universidade Federal Fluminense, \\ Niterói, RJ, Brasil. \\ ${ }^{3}$ Departamento de Engenharia Agrícola e Meio Ambiente, Universidade Federal Fluminense, \\ Niterói, RJ, Brasil.
}

Recebido 4/12/2014 - Aceito 13/5/2015

\begin{abstract}
Resumo
A seca é um fenômeno de comportamento estatístico, seus efeitos variam em função da região de estudo da relação entre a seca meteorológica (ausência de chuva) e outros fatores relacionados à seca agrícola (solos e cultivos), seca hidrológica (suprimento de água) e seca socioeconômica (impacto social e econômico). A seca meteorológica é a base para os outros tipos de seca. Neste contexto, o presente trabalho buscou analisar a intensidade da anomalia temporal do regime de chuva na microbacia de Santa Maria/Cambiocó, localizada na região Noroeste do Estado do Rio de Janeiro, Região Sudeste do Brasil. Para tanto, foi utilizado o índice de anomalia de chuva calculado com base em duas estações pluviométricas envoltórias à região com períodos consecutivos de 10 anos de dados. A estação seca, inverno, abrange os meses de junho, julho e agosto e a estação chuvosa, verão, abrange os meses de novembro, dezembro e janeiro. Não foram constatadas alterações de tendência do regime pluviométrico ao longo da série observada que pudessem influenciar a seca meteorológica. Consequentemente, não foram verificadas, no período analisado, tendências que pudessem justificar impactos de anomalias climáticas ou de mudanças climáticas ao longo das séries observadas.
\end{abstract}

Palavras chave: chuva, seca, mudança climática, Região Noroeste do Estado do Rio de Janeiro.

\section{Rain Anomaly Index Analysis for the SantaMaria/Cambiocó Catchment, Rio de Janeiro State, Brazil}

\begin{abstract}
Drought is a statistical phenomenon, its impacts vary according to the region under study and the relationship between the meteorological drought (no rainfall) and other factors related to agricultural drought (soil and crops), hydrological drought (water supply) and socioeconomic drought (social and economic impacts). Meteorological drought is the basis for the other types of drought. In this context, the present study aimed to analyse the intensity of rainfall temporal anomaly in the representative catchment of Santa Maria/Cambiocó located in the Northwest Region of Rio de Janeiro State, Southeast Region of Brazil. The rain anomaly index was calculated based in two gauge stations ten-year rainfall time series. The dry season, winter, covers the months of june, july and august and the rainy season, summer, covers the months of november, december and january. No changes were verified in the rainfall series trends that could influence the observed meteorological drought. Consequently, no tendency in the observed time period, that could be justified by climate anomalies or climate change impacts, were identified.
\end{abstract}

Keywords: rainfall, drought, climatic changes, Northwest Region of Rio de Janeiro State. 


\section{Introdução}

A ocorrência de secas como consequência de alteração climática, em várias regiões do globo terrestre, na década de setenta evidenciou a vulnerabilidade do homem a esse risco climático, demonstrando a necessidade de melhor entendimento, melhor previsão de sua ocorrência e uso correto de medidas mitigatórias. Alguns estudos sobre mudanças climáticas (e.g. Marengo, 2008; Marengo et al., 2010) têm demonstrado uma certa polarização da ocorrência das chuvas para diferentes regiões do planeta, tendendo ao aumento da ocorrência de chuvas intensas e da severidade das secas meteorológicas.

Wilhite \& Glantz (1987) enquadraram, além das secas meteorológicas, as secas como agrícolas (solos e cultivo), hidrológicas (suprimento de água) e socioeconômicas (impacto social e econômico). A seca meteorológica pode ser definida como um período constante, de um mês ou mais, durante o qual a precipitação está abaixo dos valores de precipitação média para o período.

Como aponta Cunha (2008), a definição deste tipo de seca depende da região de ocorrência, uma vez que as condições atmosféricas que resultam em deficiências de precipitação podem diferir consideravelmente de uma região para outra.

Embora as secas estejam classificadas em quatro tipos, todas são originadas da deficiência de precipitação, que resulta na falta de água para o desenvolvimento de atividades ou para a sobrevivência dos seres vivos (Wilhite, 2003).

A análise da variabilidade climática reveste-se de importância quando analisados os impactos do clima em áreas de interesse socioeconômico e ambiental, como, por exemplo, a prática inadequada de utilização do solo em bacias rurais, a partir da remoção da cobertura vegetal nativa para inserção de pastagens ou outras culturas exóticas. É através de estudos que se torna possível conhecer os problemas da região e ao mesmo tempo tentar solucioná-los ou minimizá-los por meio de planejamento de ações (Silva et al., 2010; Souza et al., 2013).

Outro fenômeno que grande impacto no clima da América do Sul é o El Niño / Oscilação Sul (ENOS) que é dividido em duas fases, a saber: El Niño (EN) e La Nina (LN). O EN e LN são eventos de grande escala, que ocorrem na Região do Pacífico Equatorial (Freire et al., 2011). O EN é um fenômeno causado pelo aquecimento das águas superficiais e subsuperficiais do Oceano Pacífico Equatorial além do normal e pela redução dos ventos alísios na região equatorial. A LN representa um fenômeno oceânico-atmosférico com características opostas ao EN, e que se caracteriza por um esfriamento anormal nas águas superficiais e subsuperficiais do Oceano Pacífico Equatorial. Segundo Blain e Kayano (2011), o EN e LN podem ser caracterizados a partir do Índice de Oscilação Sul (IOS) que é uma medida da diferença de pressão atmosférica do nível médio do mar entre a localidade de Darwin, Australia $\left(12^{\circ} \mathrm{S}\right.$ e $\left.130^{\circ} \mathrm{E}\right)$ e do Tahiti $\left(17^{\circ} \mathrm{S}\right.$ e $\left.150^{\circ} \mathrm{W}\right)$. Valores negativos do IOS caracterizam eventos de $\mathrm{EN}$, ao passo que valores positivos caracterizam episódios de LN.

Essas alterações provocam mudanças no padrão normal de circulação atmosférica, principalmente no regime pluviométrico em diversas regiões do continente. Entretanto, Minuzzi et al. (2007), a partir da análise de dados de 203 estações pluviométricas, localizadas na Região Sudeste do Brasil, verificaram que o fenômeno LN não influencia o início da estação chuvosa e, mesmo havendo anomalias de precipitação, estas pouco estiveram correlacionadas com o comportamento da temperatura da superfície do Pacífico Equatorial e, ou, IOS.

Os períodos de seca e chuva podem ser classificados por meio de índices, como o de Palmer (1965) e o de Rooy (1965). A categorização dos períodos secos e úmidos são úteis em projetos de irrigação, abastecimento de água, culturas dependentes da regularidade da chuva e em locais onde o uso de água subterrânea é pequena em relação às águas superficiais (Freitas, 2005).

No Estado do Rio de Janeiro tem dada mais importância às enchentes e suas consequências do que por impactos de secas meteorológicas. No entanto, enquanto a Região Sul do Estado apresenta altas taxas pluviométricas, maiores que $2.000 \mathrm{~mm}$ anuais, a Região Noroeste do Estado do Rio de Janeiro (RNERJ), onde se observa o desenvolvimento de atividades agrícolas significativas para o desenvolvimento econômico e social, apresentam totais anuais da ordem de 1.000 mm. Alguns municípios, como o de São José de Ubá, vêm se deparando com a degradação das paisagens, com sinais dos impactos da erosão e do empobrecimento dos solos.

A especulação sobre os impactos de anomalias de chuvas e de possíveis impactos das mudanças climáticas e seus desdobramentos nas disponibilidades hídricas para as atividades agrícolas, sociais e ambientais da RNERJ desperta a necessidade de ações por parte dos órgãos governamentais e da sociedade civil. Moraes (2007) aponta que este fato tem motivado investimento em estudos e projetos que busquem a melhor compreensão dos fenômenos climáticos e hidrológicos na região que poderão consubstanciar de forma mais efetiva as políticas públicas para a retomada do desenvolvimento sustentável local. Neste contexto, foi implementada a bacia representativa de Santa Maria-Cambiocó, no município de São José de Ubá, a escolha da área para estudos foi norteada por questões físicas e ambientais e pelos arranjos socioeconômicos locais.

Este trabalho buscou avaliar a intensidade da anomalia do regime de chuva na microbacia de Santa Maria/Cambiocó, localizada na RNERJ, utilizando o Índice de Anomalia de Chuva (IAC) proposto por Rooy (1965). Além disso, foram observados os períodos do histórico de ocorrência de EN e LN, assim como a identificação de tendências dos valores de IAC que possam ser justificadas por eventuais impactos de mudanças climáticas. 


\section{Materiais e Métodos}

A microbacia experimental de Santa Maria/Cambiocó, com área de $13,5 \mathrm{~km}^{2}$, fica localizada no município de São José do Ubá que está na parte central da RNERJ e contava com uma população de 7.003, habitantes segundo o censo IBGE de 2010. A principal atividade econômica é a agrícola e, embora existam outras culturas, o tomate constitui a base da economia local, sendo o município o segundo maior produtor de tomates do Estado do Rio de Janeiro.

A microbacia possui altitude média de $246 \mathrm{~m}$, apresenta relevo acidentado e os solos são predominantemente do tipo Latossolo. O clima é do tipo tropical semiúmido, com estação chuvosa entre outubro e abril, e estação seca entre maio e setembro, possuindo uma precipitação pluviométrica de até $1.100 \mathrm{~mm} . \mathrm{ano}^{-1}$. Um dos condicionantes climáticos configura-se na baixa altitude dos morros que não favorecem a intensificação de nuvens por forçantes topográficas, quando da incidência de frentes frias na área. O regime de temperatura é semelhante ao da Região Noroeste, variam entre $15^{\circ} \mathrm{C}$ (mínima) e $40^{\circ} \mathrm{C}$ (máxima), sendo a temperatura média $25^{\circ} \mathrm{C}$ (Moraes, 2007).

Moraes (2007) aponta que um dos aspectos importantes da escassez hídrica (seca hidrológica) na microbacia é o plantio de tomate, sobretudo nos meses críticos de estiagem quando é observada a construção de pequenos barramentos para armazenamento da água e seu uso na irrigação. Em relação à seca socioeconômica, o autor aponta que em 1999 ocorreu um evento de estiagem crítico, que atingui toda a região, causando danos às principais atividades econômicas locais (pecuária leiteira e a olericultura) e consequentemente a qualidade de vida da população formada principalmente por pequenos produtores rurais.

Para a classificação dos períodos secos e úmidos foi utilizado o IAC proposto por Rooy (1965) e posteriormente adaptado por Freitas (2005), expresso por:

$$
\mathrm{IAC}=3\left[\frac{(\mathrm{N}-\overline{\mathrm{N}})}{(\overline{\mathrm{M}}-\overline{\mathrm{N}})}\right]
$$

para anomalias positivas.

$$
\mathrm{IAC}=-3\left[\frac{(\mathrm{N}-\overline{\mathrm{N}})}{(\overline{\mathrm{X}}-\overline{\mathrm{N}})}\right]
$$

para anomalias negativas, onde $\mathrm{N}$ - precipitação mensal atual (mm.mês $\left.{ }^{-1}\right), \bar{N}$ - precipitação média mensal da série histórica (mm.mês $\left.{ }^{-1}\right), \bar{M}$ - média das dez maiores precipitações mensais da série histórica (mm.mês $\left.{ }^{-1}\right)$ e $\bar{X}$ - média das dez menores precipitações mensais da série histórica (mm.mês ${ }^{-1}$ ).

A partir dos valores encontrados, o regime de chuva foi classificado de acordo com a metodologia do IAC apresentada na Tabela 1.

O critério de seleção dos postos pluviométricos buscou identificar aqueles mais próximos, com altitudes semelhantes às da microbacia e com séries históricas de, no mínimo, dois períodos consecutivos de 10 anos.

A Tabela 2 apresenta o resumo dos postos pluviométricos selecionados, ambos localizados no Município de Itaperuna, localizado a aproximadamente $20 \mathrm{~km}$ da sede do município de São José do Ubá, extraídos dos sítios na internet do Sistema de Informações Hidrológicas (HidroWeb) da Agência Nacional de Águas (ANA) e do Instituto Nacional de Meteorologia (INMET).

A Tabela 3 apresenta as médias dos totais mensais para o período compreendido de 1942 a 2005 para o posto 004, e 1961 a 2013 para o posto 045 . Observa-se que os três

Tabela 1 - Classificação da pluviosidade segundo o IAC.

\begin{tabular}{lc}
\hline Índice de Anomalia de Chuva (IAC) & Classificação da Pluviosidade \\
\hline Maior que 4 & Extremamente Chuvoso (EC) \\
Entre 2 e 4 & Muito Chuvoso (MC) \\
Entre 0 e 2 & Chuvoso (C) \\
0 & Nem Chuvoso Nem Seco \\
Entre 0 e -2 & Seco (S) \\
Entre -2 e -4 & Muito Seco (MS) \\
Menor que -4 & Extremamente Seco (ES) \\
\hline
\end{tabular}

Tabela 2 - Postos selecionados para o estudo.

\begin{tabular}{lcccc}
\hline Código & Nome & Altitude & Período da Série Consistida & Falhas \\
\hline $02141004(004)$ & Itaperuna & $110 \mathrm{~m}$ & $03 / 1942$ a 12/2005 & $01 / 1978$ a 12/2000 \\
$02141045(045)$ & Itaperuna (INMET 83695) & $124 \mathrm{~m}$ & $01 / 1961$ a $12 / 2013$ & 01 a 05/1968; 02/1984 a 01/1992; 08 a 12/2001 \\
\hline
\end{tabular}

\begin{tabular}{|c|c|c|c|c|c|c|c|c|c|c|c|c|}
\hline \multirow[b]{2}{*}{ Posto } & \multicolumn{12}{|c|}{ Mês } \\
\hline & Jan & $\mathrm{Fev}$ & Mar & Abr & Mai & Jun & Jul & Ago & Set & Out & Nov & Dez \\
\hline 004 & 1677 & 1228 & 1140 & 741 & 360 & 247 & 196 & 174 & 405 & 1000 & 1648 & 2294 \\
\hline 045 & 1984 & 998 & 1354 & 781 & 406 & 208 & 189 & 237 & 543 & 999 & 1859 & 2270 \\
\hline
\end{tabular}

Tabela 3 - Média das precipitações mensais, em mm. 
meses consecutivos mais secos são junho, julho e agosto, sendo estes os meses definidos como o trimestre inicial para os períodos utilizados na caracterização do clima através do IAC.

Índices que quantificam a seca sob o ponto de vista meteorológico são restritos a escalas temporais maiores ou iguais à mensal. Em pequenos intervalos de tempo, a grande variabilidade temporal da precipitação dificulta o estabelecimento de um patamar esperado e consistente, que possa ser usado no cálculo de uma possível anomalia ou déficit. A tentativa de estimativa em intervalos mais curtos, como por exemplo 10 dias, usualmente adotados para avaliação da seca agrícola, resulta em valores numéricos que não apresentam correlação com parâmetros da seca meteorológica (Blain e Brunini, 2006). O período adotado para o estudo foi o trimestral.

Para o posto 004 foi possível utilizar o período contínuo de 06/1942 a 05/1977, perfazendo um total de 35 anos e permitindo o cálculo do IAC anual. O IAC trimestral foi calculado para três períodos, a saber: 06/1942 a 05/1953; 06/1953 a 05/1965; e 06/1965 a 05/1977. Já o posto 045 possui dois períodos consecutivos superiores a 10 anos que podem ser utilizados para o cálculo do IAC trimestral, a saber: 06/1968 a 05/1983 e 06/2002 a 05/2012.

Devido ao fato de nenhum dos dois postos selecionados possuírem períodos consecutivos superiores a 10 anos que abranjam a década de 1990, buscou-se utilizar os dados do posto 045 que se estendem de 06/1992 a 05/2001 (9 anos) mesmo que não seja possível caracterizar os resultados como uma normal climatológica. Nesse caso, como o posto 045 não possui série contínua próxima a 30 anos, o IAC anual não foi calculado.

Foram analisados os valores de IAC para os anos de ocorrência das anomalias climáticas de EN e LN, sobretudo os anos atribuídos como manifestações fortes desses fenômenos. Os anos de 1957-58; 1965-66; 1972-73; $1982-83$; 1987-88; $1997-98$ para a anomalia de EN; e os anos de 1973-74; 1975-76; 1988-89; 1999-00; 2010-11 para LN. Sendo que as ocorrências de 1982-83 e 1997-98 são atribuídas como as mais fortes do século XX (Smith et al., 2008; L'Heureux et al., 2012).

\section{Resultados}

A Figura 1 apresenta os valores de IAC anual para o posto 004. Nela é possível observar dois períodos de três anos consecutivos de seca: um mais fraco entre 06/1944 a 05/1947 e outro mais forte entre 06/1953 e 05/1956; e um período de três anos consecutivos com muita chuva, entre 06/1964 e 05/1967. Além disso, observam-se cinco períodos de dois anos consecutivos com bom regime de chuva e mais dois períodos consecutivos com pouca chuva. Os valores calculados para IAC anual variaram entre $+6,435 \mathrm{e}$ $-5,013$. Nesse período não foi observada tendência no aumento ou diminuição de IAC, mas verificou-se que a ocorrência de anomalias positivas foi ligeiramente maior do que as negativas.

A Figura 2 apresenta os valores de IAC trimestral para o posto 004 para o período entre 1942 e 1977. Os valores calculados para IAC trimestral variaram entre

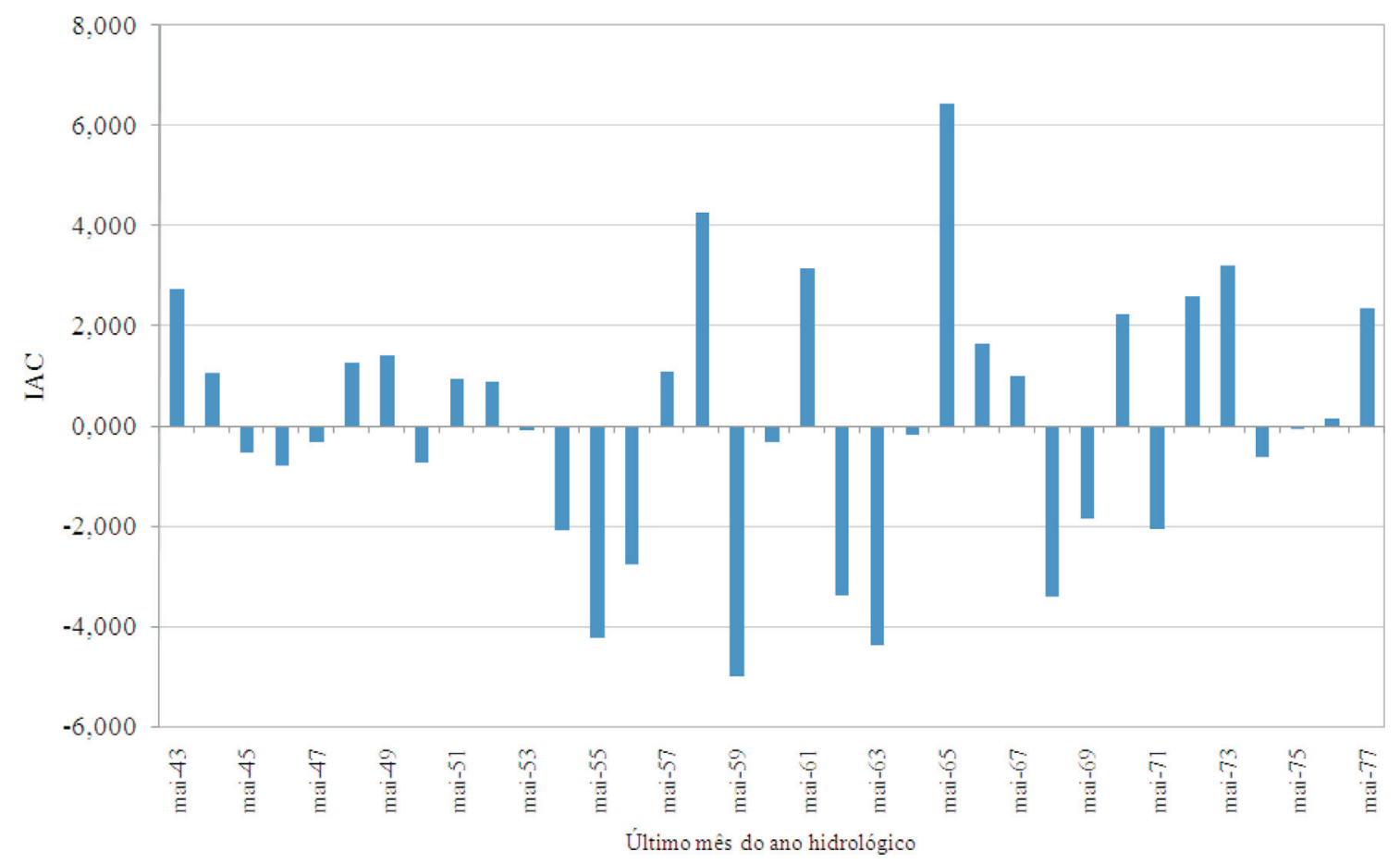

Figura 1 - IAC anual do posto 004 para o período consecutivo de 06/1942 a 05/1977. 


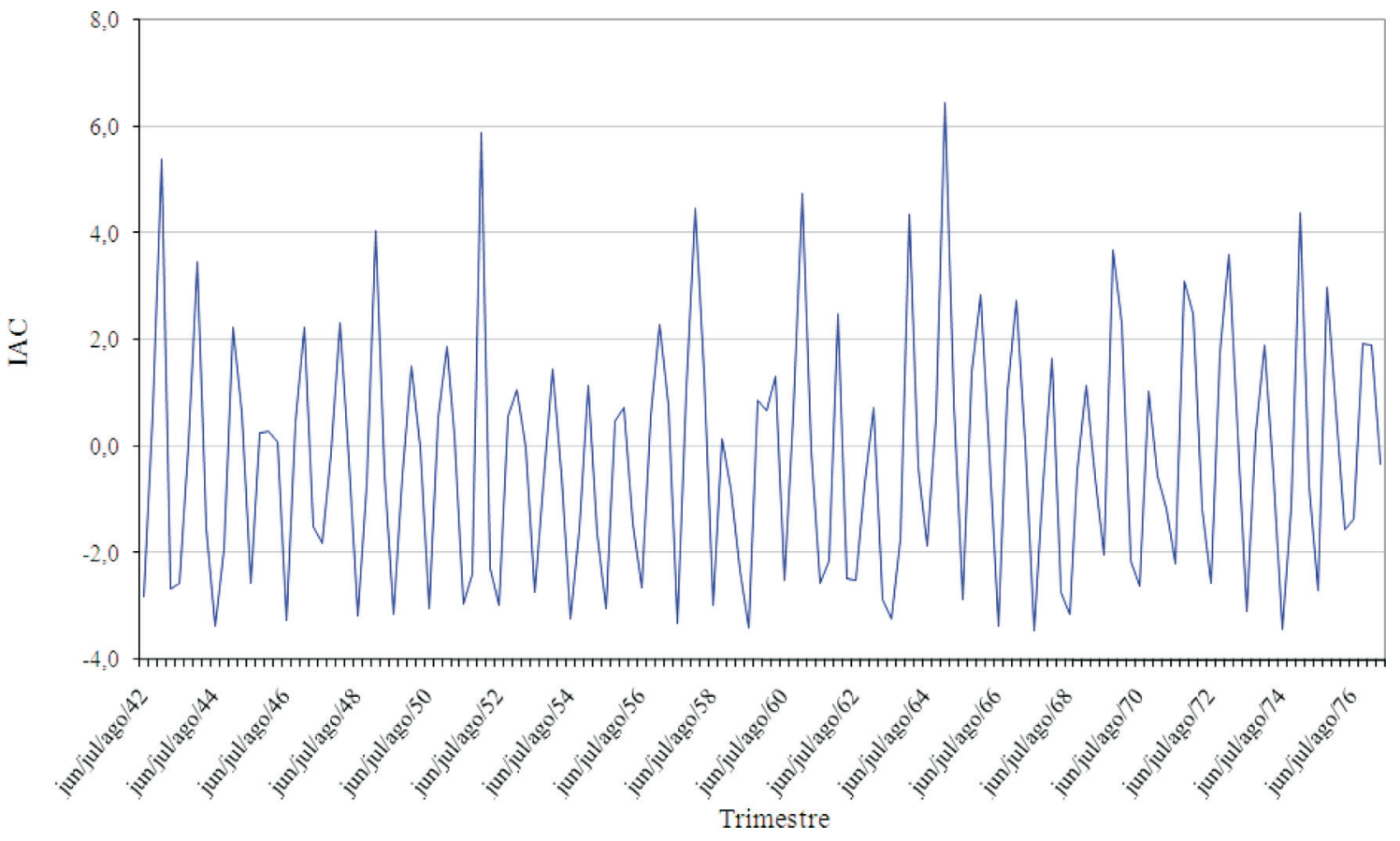

Figura 2 - IACs trimestrais para o posto 004.

$+6,441$ e -3,460. Na análise visual não se observa tendência para aumento ou diminuição, ou mesmo de sinal dos IACs.

A Figura 3 apresenta os resultados encontrados na análise de frequência das classes dos IACs trimestrais para os períodos estabelecidos no posto 004. No eixo das abscissas as siglas ES, MS, S, C, MC e EC representam as classes "extremamente seco", "muito seco", "seco", "chuvoso", "muito chuvoso" e "extremamente chuvoso", respectivamente, conforme Tabela 1.

Para o trimestre mais seco (junho, julho, agosto), em todos os períodos analisados, o mais frequente foi a classe muito seco (MS), com IACs entre $-2,0$ e $-4,0$. Para as sequências de anos mais recentes, aproximadamente 10\% dos trimestres foram enquadrados como seco (S), IAC entre 0 e -2,0. Para o trimestre de dezembro a fevereiro (chuvoso) observaram-se ocorrências da classe muito úmido (MC), IACs maiores que 4,0, mais frequente para o período entre 1942-1953. Para nenhum dos trimestres ou sequência de anos analisados foram identificados IACs na classe extremamente seco (ES), menores que -4,0.

Considerando os intervalos de anos para os quais foram determinados os IACs trimestrais, a sequência entre 1942 e 1953, foram observadas as classes entre muito seco e extremamente úmido, sendo que nessa sequência de anos foram verificados os menores valores de IAC para o trimestre mais seco, e os maiores valores de IAC para o trimestre mais úmido. A mesma tendência foi observada para o período entre 1953 e 1965 . Mas, para o período entre 1965 e 1977, embora tenha sido observado um alto percentual na classe MS, a ocorrência EC, foi significativamente menor do que para os outros períodos estudados.
A Figura 4 apresenta os valores de IAC trimestral para o posto 045 para o período entre 1962 e 2012. Os valores variaram entre $+6,049$ e $-3,503$. Assim como para o posto 004 , não foram observadas na análise visual tendências para aumento ou diminuição, ou mesmo tendência de sinal positivo ou negativo dos valores de IAC.

A Figura 5 apresenta os resultados encontrados na análise de frequência das classes dos IACs trimestrais para os períodos estabelecidos no posto 045 . As classes observadas foram entre MS (IAC entre - 2 e -4) e EC (IAC > +4), conforme Tabela 1. O trimestre mais seco, junho a agosto, foi o que registrou os maiores valores negativos em todas as sequências de anos. Os maiores valores de IAC foram registrados no trimestre mais chuvoso, entre dezembro e fevereiro, em todos os períodos analisados. Ressalta-se ainda, no período entre 1992 e 2001, a alta frequência de valores baixos de IAC, classificado como "seco" (S) no trimestre março, abril e maio, colocando em destaque esse período em relação aos demais.

Com relação à associação entre ocorrências de ENOS e os valores de IAC, a análise não foi conclusiva. Não foi observada uma única tendência, ou seja, que sempre haja mudança de regime ou aumento/diminuição de volumes nesses anos. Como mencionado anteriormente, Minuzzi et al. (2007), também não identificaram uma relação ou comportamento tendencioso forte desses fenômenos com a pluviosidade na Região Sudeste, sobretudo para a RNERJ. 

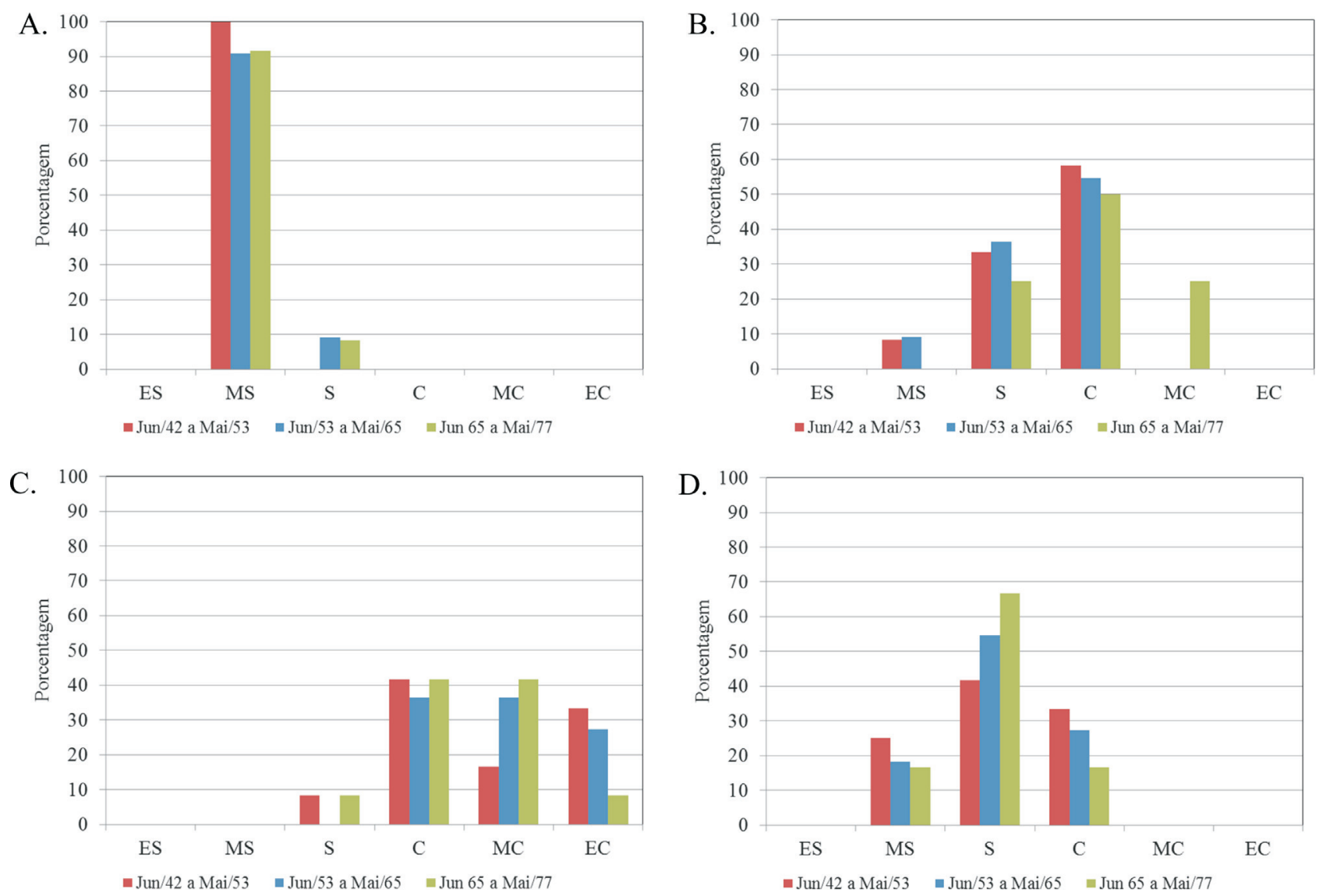

Figura 3 - Frequência das classes do IAC para o posto 004: junho, julho e agosto (A), setembro, outubro e novembro (B), dezembro, janeiro e fevereiro (C) e março, abril e maio (D).

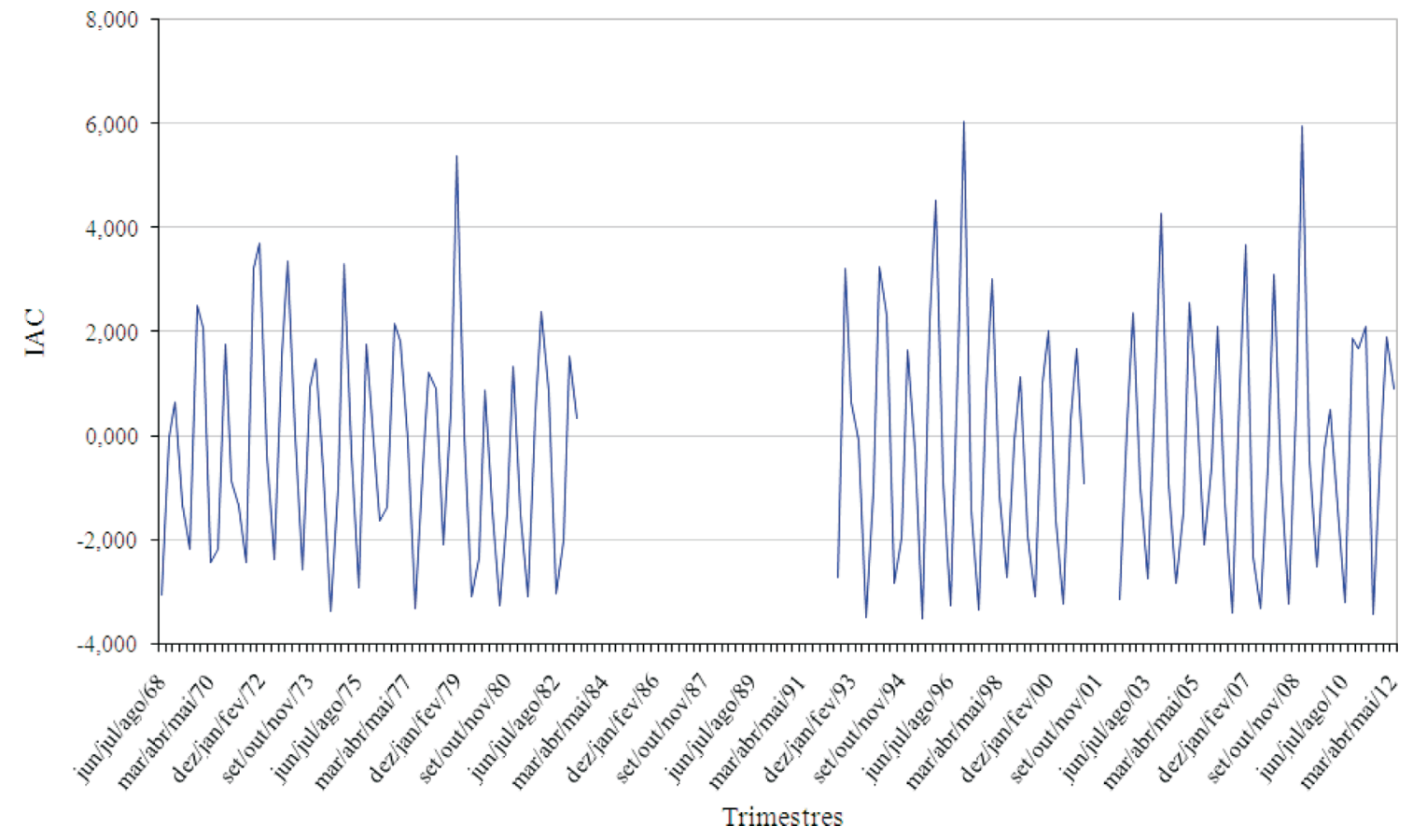

Figura 4 - IACs trimestrais para o posto 045. 

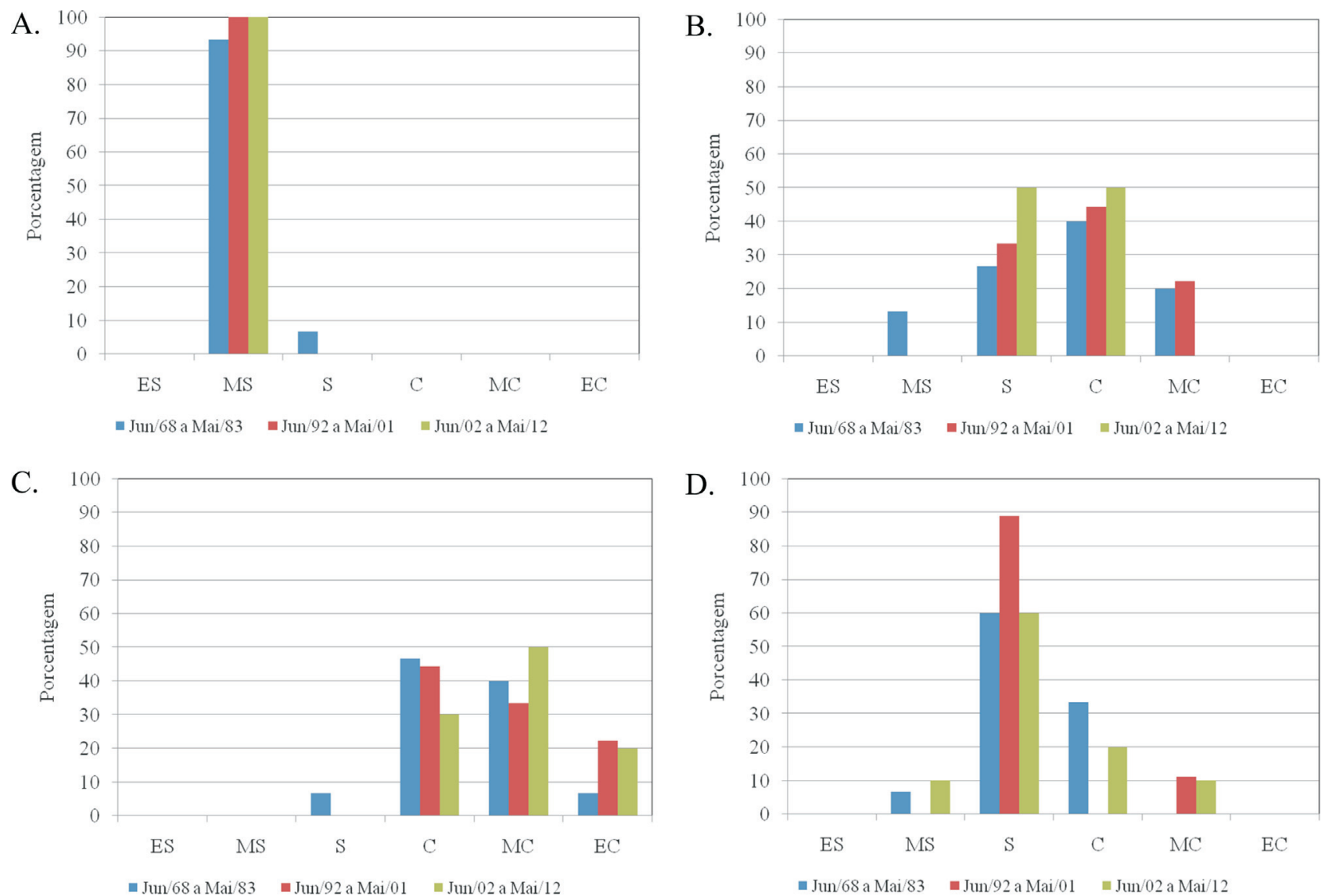

Figura 5 - Frequência das classes de IAC do posto 045: Junho, Julho e Agosto (A), Setembro, Outubro e Novembro (B), Dezembro, Janeiro e Fevereiro (C) e Março, Abril e Maio (D).

\section{Conclusões}

A estação seca na região é observada nos meses de junho, julho e agosto, e a estação chuvosa nos meses de novembro, dezembro e janeiro.

Os maiores valores absolutos do índice de anomalia de chuva são referentes às anomalias positivas. Os dois postos, em todos os períodos selecionados, apresentaram trimestres classificados como "extremamente chuvoso" e, além disso, observou-se que para cada um há um trimestre com valor superior a 6,0.

Em relação às anomalias negativas, não foi observado nenhum trimestre com valor inferior a $-4,0$, classificado como "extremamente seco". Em termos absolutos, os valores máximos negativos foram inferiores aos máximos positivos. Os resultados negativos foram próximos a $-2,0 \mathrm{em}$ trimestres consecutivos, justificando a ocorrência de chuva no período seco que atenua o pico da anomalia.

Com as informações de frequência de classes do índice de anomalia de chuva pode-se inferir que o período seco não se acentuou ao longo do tempo, como também não foi possível verificar relação entre as anomalias climáticas (El Niño / La Niña) e o índice de anomalia de chuva.

Alterações de volume precipitado que possam influenciar a seca meteorológica não foram constatados. A aridez pode estar relacionada aos fatores antrópicos, principalmente ao uso e ocupação do solo, que em conjunto com a baixa precipitação comum no inverno da região, incrementam as secas agrícola, hidrológica e socioeconômica.

\section{Referências}

BLAIN, G. C.; BRUNINI, O. Quantificação da seca agrícola pelo índice padronizado de evapotranspiração real (IPER) no estado de São Paulo. Bragantia, v.65, p.517-525, 2006.

BLAIN, G. C.; KAYANO, M. T. 118 anos de dados mensais do Índice Padronizado de Precipitação: série meteorológica de Campinas, Estado de São Paulo, Revista Brasileira de Meteorologia, v. 26, n. 1, p. 137-148, 2011.

CUNHA, R. L. A. Definição de cenários de referência para avaliação dos impactos das secas. Porto: Faculdade de Engenharia da Universidade do Porto, 2008. 147p. Dissertação Mestrado.

FREIRE, J. L. M.; LIMA, J. R. A.; CAVALCANTI, E. P. Análise de Aspectos Meteorológicos sobre o Nordeste do Brasil em Anos de El Niño e La Niña. Revista Brasileira de Geografia Física, n.3, p.429-444, 2011.

FREITAS, M. A. S. Um sistema de suporte à decisão para o monitoramento de secas meteorológicas em regiões semiáridas. Revista Tecnologia, v.suplem, p.84-95, 2005.

L'HEUREUX, M. L.; COLLINS, D. C.; HU, Z.-Z. Linear trends in sea surface temperature of the tropical Pacific Ocean and 
implications for the El Niño-Southern Oscillation. Climate Dynamics, v.40, p.1223-1236, 2012.

MARENGO, J. A. Água e mudanças climáticas. Estudos Avançados, v.22, n.63, p.83-96, 2008.

MARENGO, J.A.; RUSTICUCCI, M.; PENALBA, O.; REMON, M. An Intercomparison of observed and simulated extreme rainfall and temperature events during the last half of the twentieth century: Part 2 - historical trends. Climate Change, v.98, p.509-529, 2010 .

MINUZZI, R. B.; SEDIYAMA, G. C.; COSTA, J. M. N.; VIANELLO, R. L. Influência da La Niña na estação chuvosa da Região Sudeste. Revista Brasileira de Meteorologia, v.22, n. 3 p.345-353, 2007.

MORAES, M. F. Estimativa do balanço hídrico na bacia experimental/representativa de Santa Maria/Cambiocó Município de São José de Ubá - RJ. Rio de Janeiro: COPPE/UFRJ. 2007. 251p. Tese Doutorado.

PALMER, W.C. Meteorological drought. US Weather Bureau Res. $\mathbf{N}^{\mathbf{0}}$ 45. Washington, 58p, 1965.

SILVA, D. F.; SOUZA, F. A. S.; KAYANO, M. T. Escalas temporais da variabilidade pluviométrica na bacia hidro- gráfica do rio Mundaú. Revista Brasileira de Meteorologia, v.25, n.3, p.324-332, 2010.

SMITH, T. M.; REYNOLDS, R. W.; PETERSON, T. C.; LAWRIMORE, J. Improvements to NOAA's historical merged land-ocean surface temperature analysis (1880-2006). Journal of Climate, v.21, p 2283-2296, 2008.

SOUZA, A. B.; MELO, R. A.; SILVA, D. F. Avaliação climática e de recursos hídricos da bacia hidrográfica do Rio Jaguaribe (CE). Revista Brasileira de Geografia Física, v.6, p.1115$1140,2013$.

ROOY, M. P V. A rainfall anomaly index independente of time and space. Notes. Weather Bureau of South Africa, v.14, p.43-48, 1965.

WILHITE, D. A. DROUGHT. IN: HOLTON, J. A.; PYLE, C.; CURRY, J. A. (ed.). Encyclopedia of atmospheric science. New York: Elsevier, p.650-658, 2003.

WILHITE, D. A.; GLANTZ, M.H. Understanding the drought phenomenon: The role definations. In: Wilhite, D. A.; Easterling, W. E.; Wood, D. A. Planning for drought toward a reduction of societal vulnerability. Colorado: Westview, cap.2, p.11-14, 1987.

All the contents of this journal, except where otherwise noted, is licensed under a Creative Commons Attribution License CC-BY 